\title{
AN ELLIPTIC CURVE RANDOM NUMBER GENERATOR
}

\author{
Lap-piu Lee and Kwok-wo Wong \\ Department of Electronic Engineering, \\ City University of Hong Kong, \\ 83 Tat Chee Avenue, Kowloon Tong, HONG KONG
}

\begin{abstract}
A random number generator based on the addition of the points on an elliptic curve over finite fields is proposed. Sequences produced by this generator have passed standard statistical tests. As a result, the proposed generator is found suitable to be a random number generator.
\end{abstract}

Keywords: Elliptic Curve cryptography; Random Number Generator

\section{Introduction}

Recently, elliptic curve cryptography (ECC) has received intense scrutiny from cryptographers, mathematicians, and computer scientists around the world. The primary reason for this is its higher security over existing public key cryptographic algorithms. The best algorithm known for solving the underlying mathematical problem of ECC, referred as the elliptic curve discrete logarithm problem, takes full exponential time. On the contrary, subexponential-time algorithms are known for tackling the integer factorization and the discrete logarithm problems that RSA and DSA are relied on $[1,2]$. This implies that the algorithms for solving the elliptic curve discrete logarithm problem become infeasible much more rapidly as the problem size increases than those algorithms for the integer factorization and the discrete logarithm problems. For this reason, ECC offers a security level equivalent to RSA and DSA while using a far smaller key size [3].

On the other hand, the security of most cryptographic systems depends upon the generation of unpredictable quantities that must be of sufficient size and randomness [4]. Take ECC as an example, we need to generate random bits in order to create random curves [5]. The purpose is to preclude third parties from gaining advantage through optimizing a search strategy. This implies that we usually need to implement a random number generator in a cryptographic system. Some random number generators have been proposed $[6,7]$. However, if both the tasks of random number generation and 
encryption can be done by using the same software or hardware module, we can save hardware components, memory space and design time. This is especially important in developing applications in environment with limited resources such as smart cards. With this goal, we propose a random number generator that makes use of the basic operations required in ECC.

\section{Background of Elliptic Curve Cryptography}

We study the elliptic curve of the form

$$
y^{2}=x^{3}+a x+b .
$$

The basic operation of ECC is the addition of two points on the curve, i.e., take any two points on a specific curve, add them together, and get another point on the same curve. Suppose that $\mathrm{P}\left(x_{\mathrm{P}}, y_{\mathrm{P}}\right)$ and $\mathrm{Q}\left(x_{\mathrm{Q}}, y_{\mathrm{Q}}\right)$ are two points on the same elliptic curve. To find $\mathrm{R}\left(x_{\mathrm{R}}, y_{\mathrm{R}}\right)=\mathrm{P}+\mathrm{Q}$, the following formula are used.

$$
\begin{aligned}
& x_{\mathrm{R}}=s^{2}-x_{\mathrm{P}}-x_{\mathrm{Q}} \\
& y_{\mathrm{R}}=-y_{\mathrm{P}}+s\left(x_{\mathrm{P}}-x_{\mathrm{R}}\right)
\end{aligned}
$$

where

$$
s=\left(y_{\mathrm{P}}-y_{\mathrm{Q}}\right) /\left(x_{\mathrm{P}}-x_{\mathrm{Q}}\right) \text {, if } \mathrm{P} \neq \text { ? Q }
$$

or

$$
s=\left(3 x_{\mathrm{P}}^{2}+a\right) /\left(2 y_{\mathrm{P}}\right) \text {, if } \mathrm{P}=\mathrm{Q} .
$$

Note that if $\mathrm{Q}$ is the negative point of $\mathrm{P}$, i.e., $\mathrm{Q}=-\mathrm{P}\left(x_{\mathrm{p}},-y_{\mathrm{p}}\right)$, then $\mathrm{P}+\mathrm{Q}=$ $\boldsymbol{O}$. The additive identity $\boldsymbol{O}$ is the point that gives the same point back when added to any point on the curve. This special point is called the "point at infinity". When $\mathrm{P}=\mathrm{Q}, \mathrm{R}=2 \mathrm{P}$ and we call this the "double" operation [8].

Multiplying an integer $k$ to a point $\mathrm{P}$ on an elliptic curve is equivalent to adding $\mathrm{P}$ to itself for $(k-1)$ times. This is referred as the " $k \mathrm{P}$ " operation. For an integer $r$, if $r \mathrm{P}=\boldsymbol{O}$, then $r$ is the order of $\mathrm{P}$. By converting $k$ into binary form, make use of Koblitz's trick [9], and then use the addition and double rules of elliptic curve, the $k \mathrm{P}$ operation can be done rapidly.

In practice, we only use elliptic curves over finites fields [10] for cryptography. Since our aim is to integrate a random number generator in a cryptographic system, only finite fields are concerned. These fields include prime integer, prime polynomial and normal basis fields. In this paper, prime integer finite field is used for easy understanding. The formulas stated above do not need to be modified substantially. Instead, we apply all rules end with reduction modulo a large prime number $p$, the size of the finite field [11]. 


\section{The Proposed Generator}

A block diagram of the proposed generator is shown in Figure 1 . The $k_{n} \mathrm{P}$ module is used to perform the $k \mathrm{P}$ operation, in the $n^{\text {th }}$ cycle, on a high-order point $\mathrm{P}$ over a non-supersingular elliptic curve $\mathrm{E}$. Initially, we need to choose $\mathrm{E}, p, \mathrm{P}$, and the seed $k_{1}$. The length of $k_{1}$ depends on the size of the finite field and its value should not be equal to the order of $\mathrm{P}$ over that finite field. This prevents $k_{1} \mathrm{P}$ be the point at infinity.

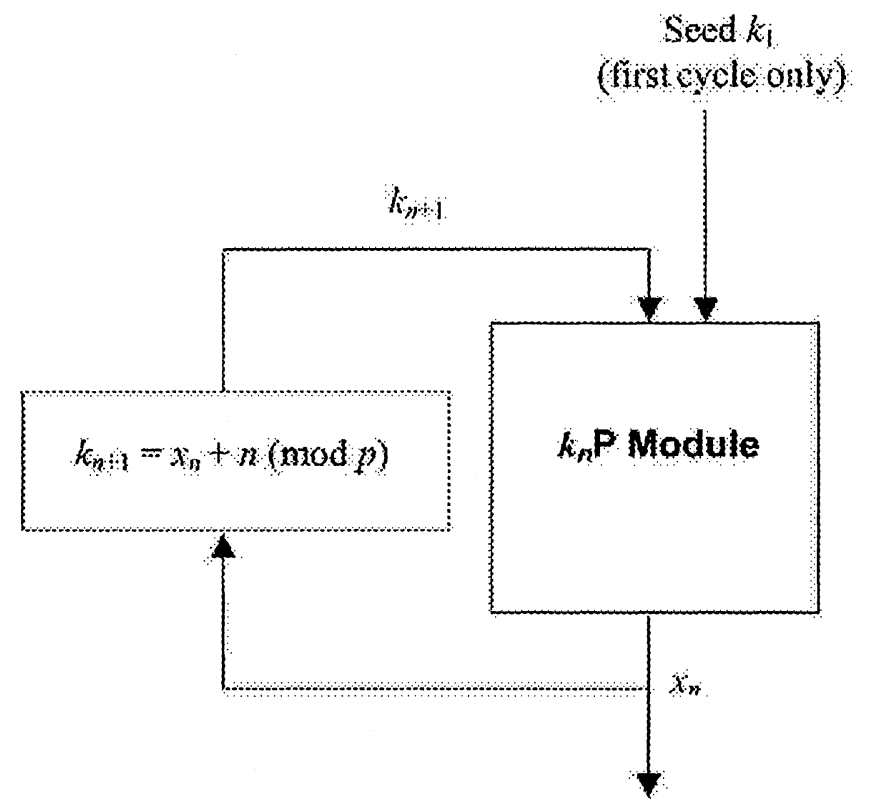

Bit Sequenee Output

Figure 1. Block diagram of the proposed random number generator

After $k_{n}$ is fed into the $k_{n} \mathrm{P}$ module, a point $k_{n} \mathrm{P}$ is generated. We use the sum of the current cycle number and the $x$-coordinate of $k_{n}$ P, i.e., $\left(x_{n}+n\right)$ modulo the selected field size $p$ as the new seed $k_{n+1}$ for the next cycle. When this operation is done recursively, a sequence of bits can be obtained by collecting the $x_{n}$ s. However, there is still a chance (although very small) that $k_{n} \mathrm{P}$ is the point at infinity. If this situation occurs, we will discard that $k_{n} \mathrm{P}$ and then re-generate it by setting $k_{n}=y_{n-1}+n-1(\bmod p)$ instead of $k_{n}=x_{n-1}$ $+n-1(\bmod p)$. For the easy understanding of the flow of the random number generation, this special case is not shown in Fig. 1.

For security reason, we should not include all the bits from the output of the $k_{n} \mathrm{P}$ module in the random bit sequence. Instead, we choose the output 
bits selectively by a simple bit selection module. This module selects the bits at an interval equal to the current cycle number $n$ modulo the bit length of the field size. For instance, if the current cycle is $168(n=168)$ and the bit length of the field size is 163 , then the bits at position $5,10,15,20 \ldots 160$ are selected.

\section{Period of the Generator}

The purpose of setting $k_{n+1}=x_{n}+n(\bmod p)$ is to increase the period of the generator. If $n$ is not added to $x_{n}$, the bit sequence depends solely on the output of the $\mathrm{kP}$ operation. It will start to repeat itself when there exists a $x_{n-i}$ $(1<i<n)$ such that $x_{n}=x_{n-i}$. For example, if the $x$-coordinate of $10 \mathrm{P}$ is 13 and a pattern: $13,3,10$ was generated in the past 3 cycles, this pattern will repeat itself in the subsequent cycles. However, if $n$ is added to $x_{n}$, the period will be governed by the field size $p$. This property is shown by the following analysis.

$$
\begin{aligned}
& \text { In the } s^{\text {th }} \text { cycle, } \\
& \quad k_{s+1}=x_{s}+s(\bmod p),
\end{aligned}
$$

where $x_{s}$ is the output of the $k_{n} \mathrm{P}$ module shown in Fig. 1.

In the $t^{\text {th }}$ cycle,

$$
k_{t+1}=x_{t}+t(\bmod p)
$$

where $x_{t}$ is the output of the $k_{n} \mathrm{P}$ module, $t>s$.

Suppose the output of the $k_{n} \mathrm{P}$ module of the $t^{\text {th }}$ cycle is the same as that of the $s^{\text {th }}$ cycle. That is,

$$
x_{t}=x_{s} \text {. }
$$

If the output of the $k_{n} \mathrm{P}$ module of the $(t+1)^{\text {th }}$ cycle is also equal to that of the $(s+1)^{\text {th }}$ cycle, then $k_{t+1}$ must equal to $k_{s+1}$. By (6) and (7), we have,

$$
x_{t}+t=x_{s}+s(\bmod p) \text {. }
$$

By (8), (9) becomes,

$$
t=s(\bmod p) \text {. }
$$

Since $t>s$, we have $t=s+m p$, where $m$ is a non-zero positive integer. Hence the output pattern will repeat after $p$ cycles. Nowadays, field sizes over 160 bits are usually used in practical ECC system. As a result, $p \sim 2^{160}$ and 160 bits are generated in each cycle. If there is not the bit selection module, the maximum period of the proposed generator is about $160 \times 2^{160}$ bits. Even when it exists and only allows one bit as output in each cycle, the 
period is still about $2^{160}$ bits. Therefore, the proposed generator is able to generate random number sequences with long periods.

\section{Tests and Results}

Besides the period, another important issue that we are concerned about a random number generator is the distribution of the bits generated [12]. The output sequence of the generator has to go through standard statistical tests as specified in FIPS 140-2 [13] for a test of randomness. There are a total of 4 tests. The first one is the monobit test, which is used to examine the balance of the single bits, 0 's and ' 1 's. The next one is the poker test. It is for testing the number of occurrences of each of the 16 possible 4-bit combinations. The third one is the runs test that aims at checking various consecutive occurrences of $1 \mathrm{~s}$ or $0 \mathrm{~s}$. The last test (long run test) is used for testing whether the maximum consecutive occurrence is over a tolerance limit. A single bit stream of output from the generator is subjected to the tests. If it fails in any of the tests, the generator is rejected from being considered as a random number generator.

We use the P-163 elliptic curve chosen by Certicom Corporation [14] for our experiment. The parameters of the curve as well as the finite field size are listed in hexadecimal form as follows.

Curve Parameter $a=04$ 3182D283 FCE38807 30C9A2FD D3F60165 $29 \mathrm{~A} 166 \mathrm{AF}$

Curve Parameter $b=02$ 0C61E945 9E53D887 1BCAADC2 DFC8AD52 25228035

Field Size $p=05$ 177B8A2A 0FD6A4FF 55CDA06B 0924E125 F86CAD9B

Three pairs of the point $\mathrm{P}$ and the seed $k_{1}$ are selected arbitrarily for generating three different bit sequences. Their values are listed as follows.

Case 1:

$\mathrm{P}\left(x_{\mathrm{P}}, y_{\mathrm{P}}\right)=(00$ 17E70122 77E1B4E4 3F7BF746 57E8BE08 BACA175B, 00 AA03A0A8 26907046 97E8C504 CB135B2B 6EEF3C83)

$k_{1}=03$ 177B8A2A 0FD674FF 556AA7B8 A7851F88 BD53B2C1

Case 2:

$\mathrm{P}\left(x_{\mathrm{P}}, y_{\mathrm{P}}\right)=(00$ 17E70122 77E1B4E4 3F7BF746 57E8BE08 BACA175B, 00 AA03A0A8 26907046 97E8C504 CB135B2B 6EEF3C83)

$k_{1}=01$ 1537AB48 397E4CD4 37F23A9C A23C9B75 455552FD

Case 3:

$\mathrm{P}\left(x_{\mathrm{P}}, y_{\mathrm{P}}\right)=(01 \mathrm{DC1E} 9 \mathrm{~A} 48$ 2085B3DF A722EB7A 541D5050 5ED31DCA, 01 2D71ECC1 578BFBE2 03D0C2CE 238EB606 0ADCAA1E) $k_{1}=02$ 4563ED51 81DBC76A 31EFDC45 96AC5B6A 7EFC45B0 
The testing results for these three sequences are listed in Table 1. They show that all the three sequences have passed the four tests. As a result, the proposed generator can be accepted as a random number generator.

Table 1. Testing results of the three output sequences

\begin{tabular}{|c|c|c|c|c|}
\hline \multirow[b]{2}{*}{$\begin{array}{c}\text { Statistical } \\
\text { test }\end{array}$} & \multirow[b]{2}{*}{ Required interval } & Case 1 & Case 2 & Case 3 \\
\hline & & $\begin{array}{c}\text { Output } \\
\text { value } \\
(X)\end{array}$ & $\begin{array}{l}\text { Output } \\
\text { value } \\
(X)\end{array}$ & $\begin{array}{c}\text { Output } \\
\text { value } \\
(X)\end{array}$ \\
\hline Monobit test & $9725<X<10275$ & 9827 & 9929 & 9835 \\
\hline Poker test & $2.16<X<46.17$ & 23.1456 & 12.0160 & 21.3536 \\
\hline Runs test & $\begin{array}{ll}\text { Run }=1, & 2343 \leq X \leq 2657 \\
\text { Run }=2, & 1135 \leq X \leq 1365 \\
\text { Run }=3, & 542 \leq X \leq 708 \\
\text { Run }=4, & 251 \leq X \leq 373 \\
\text { Run }=5, & 111 \leq X \leq 201 \\
\text { Run } \geq 6, & 111 \leq X \leq 201\end{array}$ & $\begin{array}{c}2546 \\
1251 \\
597 \\
330 \\
146 \\
138\end{array}$ & $\begin{array}{c}2529 \\
1226 \\
641 \\
324 \\
156 \\
138\end{array}$ & $\begin{array}{c}2579 \\
1191 \\
613 \\
325 \\
133 \\
150\end{array}$ \\
\hline Long run test & Run $\geq 26, \quad X=0$ & 0 & 0 & 0 \\
\hline Final Result & & PASS & PASS & PASS \\
\hline
\end{tabular}

\section{Discussion}

In this new approach, the number of output bits is no longer fixed. It can be determined by the number of operation cycles. Moreover, by simply changing the seed $k_{1}$ and the initial point $\mathrm{P}$, a different bit sequence can be generated. However, these two parameters should be kept secret for security. Basically, $\mathrm{P}$ can be any point on the elliptic curve. However, if we implement this generator with ECC together, P should not be the point that used in the ECC part. This is because the points used in ECC are always treated as the parameters of public keys.

There is a trade-off between the efficiency in bit sequence generation and the security level. The bit selection module is actually very flexible. For maximum security, we can select just one bit in each cycle, but the efficiency will be degraded. On the other hand, if a fast generation is required, we can select more bits as the output, but the security level is lower.

As mentioned above, elliptic curve operations can be implemented not only in prime integer fields, but also in other finite fields. Since the operations can be done efficiently over optimal normal basis (only AND, XOR, and ROTATE operations are needed [5]), the proposed random number generator is suitable to be implemented in hardware.

\section{Conclusion}

In this paper, a new approach for constructing a random number generator using basic operations in ECC is presented. The period of the bit sequences is 
analyzed theoretically. Moreover, bit sequences generated randomly have passed the standard tests and so they possess a satisfactory degree of randomness. Nowadays, ECC is accepted as a mature technology and there will be an increasing number of cryptographic processors and software specially designed for it. Since our proposed random number generator is based on the core operation of ECC, it can be designed and implemented efficiently using the existing components.

\section{References}

[1] J.Crowie, B.Dodson, R.Elkenbracht-Huizing, A.Lenstras, P.Montgomery and J.Zayer, A World Wide Number Field Sieve Factoring Record: on to 512 bits, in: Advances in Cryptology - ASIACRYPT 96 ( Springer-Verlag, 1996) 382394

[2] D.Gordon, Discrete Logarithms in $G F(p)$ Using the Number Field Sieve (SIAM Journal on Discrete Mathematics, vol. 6, 1993) 124-138

[3] B.Schneier, Applied Cryptography, 2nd ed. (John Wiley \& Sons, New York, 1996)

[4] O.Goldreich, S.Goldwasser and S.Micali, How to Construct Random Functions (Journal of Association for Computing Machinery, vol. 33, no. 4, 1986) $792-807$

[5] M.Rose, Implementing Elliptic Curve Cryptography (Manning Publications Co., Greenwich, 1999)

[6] Jorge A.Gonzalez, Ramiro Pino, A random Number Generator Based on Unpredictable Choatic Functions, Comput. Phys. Commun. 120 (1999) 109144

[7] Lev N.Shchur, On the Quality of Random Number Generator with Taps, Comput. Phys. Commun. 121-122 (1999) 83-85

[8] A.J.Menezes, Elliptic Curve Public Key Cryptosystems (Kluwer Academic Publishers, Boston, 1993)

[9] N.Koblitz, CM - Curves with Good Cryptographic Properties in: CRYPTO 91 (Springer-Verlag, New York, 1997)

[10] R.Lidl and H.Niederreiter, Introduction to Finite Fields and Their Applications (Cambridge University Press, Cambridge, England, 1994)

[11] F. Morain, Building Cycle Elliptic Curves Modulo Large Primes, in: EUROCRYPT 91 ( Springer-Verlag, Berlin, 1991) 328-336

[12] Ana Proykova, How to Improve a Random Number Generator, Comput. Phys. Commun. 124 (2000) 125-131

[13] Cryptographic Standard and Validation Programs at NIST, http://csrc.nist.gov/cryptval/

[14] Certicom, http://www.certicom.com 